\title{
Anorexia as a Symptom of Sexual Abuse
}

\author{
Antonio Cervero Fernández-Castañón* \\ University of Oviedo, Spain
}

*Corresponding author: Antonio Cervero Fernández-Castañón, University of Oviedo, Plaza de Feijoo, 33003 Oviedo, Spain, Tel: 665455158;

Email: cerveroantonio@uniovi.es

Submission: 眥 May 14, 2018; Published: 些 May 16, 2018

Abbreviations: BDI: Beck's Depression Inventory; BSS: Beck's Suicide Scale; BSQ: Body Shape Questionnaire; EDI: Eating Disorder Scale

\section{Opinion}

Within psychology, the evaluation of a case that allows us to establish a primary diagnosis generally involves setting intervention and treatment guidelines, which are more or less standardised and must be adapted to the patient's individual needs. In spite of this, sometimes getting new information can let us appreciate that a primary disorder is, in fact, a secondary symptom stemmed from a previous original situation which is necessary to know in order to solve the original causes that sustain it. Bearing this in mind, we will look at the real case of a patient, whose anorexia was actually a symptom derived from a disguised condition of gender-based violence that she was suffering at home.

Based on the number of sufferers, anorexia nervosa has traditionally been catalogued as one of the main eating disorders, although it can share this with bulimia nervosa, which is a disorder with a similar number of sufferers, maybe more, and with which it has a close relationship that, sometimes, creates problems when it comes to a differential diagnosis, being normal that a sufferer may alternate anorexic and bulimic patterns, making the diagnosis difficult depending on the moment of evaluation. In fact, it is estimated that the anorexia prevalence is around 1\% worldwide, even though this datum requires to consider its variability according to factors such as the economic development of societies (it is more frequent in developed societies), the sufferers' age group (it is especially relevant between 18 and 25 years old), gender (it affects mainly women but the proportion of men is increasing) or even the work environment (it is more frequent within the fashion, cinema or sport fields).

Traditionally, anorexia has been described as a defined primary disorder based on categorical pathological taxonomies that identify it because of specific characteristics among which we can highlight: body image distortion, distorted perception of the proprioceptive stimuli and self-confidence deficit regarding their capabilities and abilities, which result in a personal inefficiency feeling. All of this generates its most defining and visible characteristic, that is, the voluntary decrease in food intake, although it will have to be previously established which ones are the predisposing, precipitating and maintenance factors that are operating on the specific case study. Once we have established the correct anorexia diagnosis, it is essential to consider the most relevant individual factors in order to be able to work on them.

Although in these cases the norm is to develop the intervention and treatment based on programs that are already standardised, the cognitive behavioural therapy, which should be accompanied by suitable medical and nutritional control, even in cases that do not require hospitalisation such as the onewe are going to analyse, should include: psycho-educational methods about the characteristics of the disorder in order to reduce or remove the dysfunctional beliefs about nutritional aspects and the disorder itself, both the developed behaviour and its consequences; behavioural strategies and methods such as the behavioural contract that encourages motivation and commitment to the process, the self-registration for the establishment of the base line and the progress assessment, and the adjustment patterns of the eating behaviour like stimuli control that allow to normalise and regulate the frequency and the food intake both quantitatively and qualitatively, methods such as cognitive restructuring to alleviate the effects of the cognitive distortions, the feeling of inefficiency and low self-esteem: anxietyreducing techniques such as Jacobson's progressive relaxation or diaphragmatic breathing in order to avoid projecting the excess of activation towards food, and even working on social skills in order to face tangential aspects of an environmental disorder.

In this specific case, A.C.S. is a 45-year-old patient that visited my practice because she was in a depressive mood which manifested itself in apathetic behaviour, feeling useless and clumsy, frequent crying and, even occasional suicidal thoughts. First she did not mention any family or work problems or any eating behaviour changes, although there were visible external signs. She had never 
sought psychological help before, mentioning that her family was not keen on going to this type of services and being especially reluctant to go to the psychiatrist to assess appropriateness of a possible pharmacological treatment.

During the evaluation period that took place in the first sessions a clinical interview was carried out and evaluation questionnaires centred on depression, such as Beck's Depression Inventory (BDI) or Beck's Suicide Scale (BSS), were applied, uncovering serious symptoms of depression. During the third session cuts in her wrists can be seen, the patient claiming she had cut herself with a razor, something which she used to do "when she was a teenager and that helped her to reduce the anxiety that was caused by an eating problem she had had when she was young, but that was under control now."

During the following sessions when we work mainly on depressive symptoms, she recounts that her mother used to impose very strict diets on her because she was obsessed with weight and her family educational patterns regarded not putting on weight as something which was very important, although she is very reluctant to talk about the issue of food. During the ninth session, three months after the beginning of the treatment, she talks openly about this type of problems, saying that she finds eating very hard and feels less anguish if she does not eat, as it gives her a "feeling of disappearing", stating that in this way "she draws less attention to herself and she is seen less, having more room to lose weight." She also recounts important deterioration signs because of eating restrictions which have been happening for some time, such as: fainting, jaundice, choking feeling when eating...

The psychological evaluation starts and focuses on eating implementing tests like Eating Attitudes towards Food Test (EAT-
40) Body Shape Questionnaire (BSQ) and Eating Disorder Scale (EDI), moreover a full medical evaluation is requested, diagnosing anorexia nervosa and starting psychological intervention from a cognitive behavioural point of view, including previously mentioned techniques and complementing it with pharmacological treatment consisting of Mirtazapine and Alprazolam.

Three months after the beginning of the treatment of the eating disorder, in which improvements were scarce, we begin to suspect the existence of a withheld situation of gender -based violence, continuous sexual abuse and occasional assaults that had been happening for years. Getting this new information enabled us to deal with this new problem and to rethink the cognitive treatment which stemmed from it, so that the patient seemed to be introjecting the need that her environment had over her body, as well as internalising certain feeling of guilt over the abuse (especially the sexual one) because of being a woman. In one of the sessions she even mentioned that she wished for her female condition to disappear to prevent the abuser from feeling attracted to her, trying to escape the sexual abuse. The cognitive work meant a change when assessing the anorexia nervosa with a progressive recovery and nearly completes a year later at the present time.

\section{References}

1. Belloch A Sandín B, Ramos F (2008) Manual de Psicopatología (vol. I), McGraw Hill, Madrid, Columbus, USA.

2. Galsworthy-Francis L, Allan S (2016) Terapia cognitivo-conductual para la anorexia nerviosa: una revisión sistemática. RET: Revista de toxicomanías (76): 25-51.
Creative Commons Attribution 4.0 International License

For possible submissions Click Here

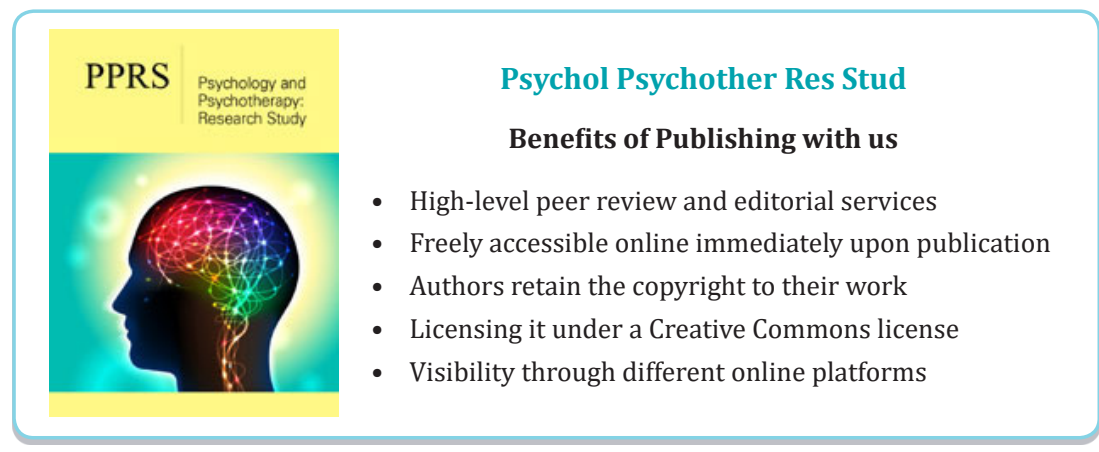

